\title{
Optimal control strategy for energy saving in trains under the four-aspect fixed autoblock system
}

\author{
Qiheng $\mathrm{LU}^{1 *}$, Xiaoyun FENG ${ }^{2}$ \\ 1. School of Electrical Engineering, Southwest Jiaotong University, Chengdu 610031, China \\ 2. School of Postgraduate, Southwest Jiaotong University, Chengdu 610031, China
}

\begin{abstract}
This paper deals with both the leading train and the following train in a train tracking under a four-aspect fixed autoblock system in order to study the optimum operating strategy for energy saving. After analyzing the working principle of the four-aspect fixed autoblock system, an energy-saving control model is created based on the dynamics equation of the trains. In addition to safety, energy consumption and time error are the main concerns of the model. Based on this model, dynamic speed constraints of the following train are proposed, defined by the leading train dynamically. At the same time, the static speed constraints defined by the line conditions are also taken into account. The parallel genetic algorithm is used to search the optimum operating strategy. In order to simplify the solving process, the external punishment function is adopted to transform this problem with constraints to the one without constraints. By using the real number coding and the strategy of dividing ramps into three parts, the convergence of GA is accelerated and the length of chromosomes is shortened. The simulation result from a four-aspect fixed autoblock system simulation platform shows that the method can reduce the energy consumption effectively in the premise of ensuring safety and punctuality.
\end{abstract}

Key words: leading train; following train; four-aspect fixed autoblock system; optimal control strategy of energysaving; train tracking; dynamic speed constraints; genetic algorithm

(C) 2011 JMT. All rights reserved.

\section{Introduction}

$\mathrm{R}$ ailway transportation departments, depending on energy heavily, have a responsibility to save energy. Normally, train diagrams allow drivers to select different operating strategies, which correspond to different energy consumption.

A number of studies have been conducted to optimize train operating strategies for saving energy. With the help of genetic algorithm (GA), and using the control of coasting position, Chang and Sim [1] optimized the operating strategy of subway trains. In [2-5], the problem was solved by using the tool of K-T condition in optimal control theory, and the optimal control model for saving energy was built based on train control notch. By analyzing the structure of a typical section, Jin et al. [6] proposed an optimization method of train operation for saving energy based on GA. Then Fu [7] put forward a strategy of operating a following train for saving energy based on the cellular automaton model.

Received Mar. 15, 2011; revision accepted May 17, 2011 *Corresponding author. Tel.: +86-13683435672; E-mail: luqiheng@gmail.com (Q.H. LU)

doi: 10.3969/j.issn.2095-087X.2011.02.002
However, [1-6] all focused on a single running train; [7] aimed at the following train in a train tracking, and did not involve the leading train. In fact, every train running on a real line is interfered not only by the line conditions, but also by its leading train, whose interference is represented by the control of the signal system. Unlike that of the line conditions, the interference from the leading train is dynamic. Compared with the studies on a single train, the research targeted at both of the leading train and following train is more complicated. With the help of GA, this paper deals with the leading and the following trains in a train tracking and an optimum operating strategy is proposed for the two trains, based on the optimal operating model of trains for saving energy.

\section{Four-aspect fixed autoblock system}

The working principle of the four-aspect fixed autoblock system is illustrated in Fig. 1, with two features. One is that the signals have four aspects, which can predict the block status of three blocks forward. The other is that there are three speed grades and the braking distance from the specified speed to 0 requires two blocks. 


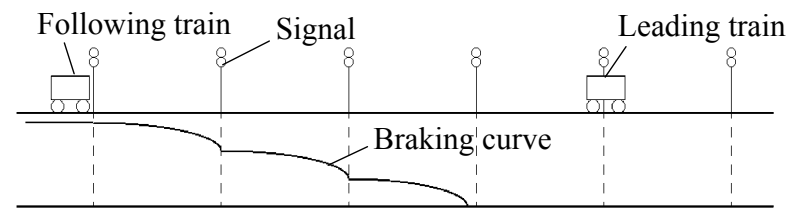

Fig. 1 Working principle of the four-aspect fixed autoblock system

\section{Model}

\subsection{Description of the problem}

Under the control of the signal system, the leading train and the following train depart in turn. After finishing their trip, they all arrive at the destination and stop. The energy consumption of the leading train and the following train are $\boldsymbol{Q}_{1}$ and $\boldsymbol{Q}_{2}$, respectively. Let $f\left(\boldsymbol{X}_{i}\right)=\boldsymbol{Q}_{i}$ $(i=1,2)$, and then the object function is demonstrated as

$$
\min \left(f\left(\boldsymbol{X}_{1}\right)+f\left(\boldsymbol{X}_{2}\right)\right) .
$$

The control variables of the function $f\left(\boldsymbol{X}_{i}\right)$ are the train control notch and the train position when its control notch is changed. Because the control notch could be changed many times during the whole trip, the variable $\boldsymbol{X}_{i}$ in (1) should be a two-dimensional matrix as

$$
\boldsymbol{X}_{i}^{\mathrm{T}}=\left(\boldsymbol{x}_{1}, \boldsymbol{x}_{2}, \cdots, \boldsymbol{x}_{n}\right)=\left(\begin{array}{l}
l_{1}, l_{2}, \cdots, l_{n} \\
p_{1}, p_{2}, \cdots, p_{n}
\end{array}\right),
$$

where $\boldsymbol{x}_{j}(j=1,2, \ldots, n)$ is the element of matrix $\boldsymbol{X}_{i}$, which include the control notch $l_{j}$ and the train position $p_{j}$. Thus, (1) is transformed into

$$
\min \left(f\left(\boldsymbol{X}_{1}^{\mathrm{T}}\right)+f\left(\boldsymbol{X}_{2}^{\mathrm{T}}\right)\right)
$$

\subsection{Dynamic equations of the trains}

The running trains are affected by the traction, resistances, and braking forces, which result in different trip time and energy consumption. The dynamic equations of the trains are as follows:

$$
\begin{aligned}
& a=\frac{\left(f_{(v)}-r_{(v)}-p_{(s)}\right) \times 9.8}{1000} \\
& r_{(v)}=a+b v+c v^{2} \\
& B=B_{k}+B_{d}=\sum\left(\varphi_{h} \sum K_{h}\right)+B_{d}, \\
& Q_{(T)}=\int_{0}^{T} Q_{y(t)} \mathrm{d} t=\int_{0}^{T} \frac{U_{w} \sum\left[\left(I_{p 2}+I_{p 0}\right) \Delta t\right]}{60} \mathrm{~d} t,
\end{aligned}
$$

where $a$ is acceleration, in $\mathrm{m} / \mathrm{s}^{2} ; f(v), r(v)$ and $p(s)$ are unit driving force, basic resistance and gradient resistance, respectively, in $\mathrm{N} / \mathrm{kN} ; a, b$ and $c$ are the coefficients of basic resistance; $B$ is the common brake force of the train; $B_{k}$ is air brake force and $B_{d}$ electric brake force, in $\mathrm{kN} ; \varphi_{h}$ is the conversion friction coefficient; $\sum K_{h}$ is the total conversion brake shoes pressure of the train, in $\mathrm{kN} ; Q_{T}$ is the total energy consumed by the train, in $\mathrm{kW} \cdot \mathrm{h} ; Q_{y(t)}$ is the energy consumed by the train at time $t$, in s; $T$ is the trip time, in s; $U_{W}$ is the voltage of train pantograph, in $\mathrm{kV} ; I_{P 0}$ is the active current of the locomotive devices, in $\mathrm{A}$; and, $I_{P 2}$ is the average active current of the locomotive under partial load, in A.

\subsection{Constraints}

\subsubsection{Boundary constraints}

The boundary constraints of trip time and speed are

$$
\left.\begin{array}{l}
t_{(0)}=0, t_{(X)}=T, \\
V_{(0)}=0, V_{(X)}=0,
\end{array}\right\}
$$

where $t_{(0)}$ and $V_{(0)}$ are, respectively, the time and speed of the train when departing; and $t_{(X)}$ and $V_{(X)}$ are, respectively, the time and speed of the train arriving at the destination.

\subsubsection{Static speed constraints}

The static speed constraints are defined by the line conditions, including the curve speed limits, the tunnel speed limits, the turnout speed limits, etc., demonstrated as

$$
V_{\max _{-} s}=\left\{\begin{array}{cl}
V_{\max _{-} s 1}, & 0 \leq s<S_{1}, \\
V_{\max _{-} s 2}, & S_{1} \leq s<S_{2}, \\
\cdots & \\
V_{\max _{-} s n}, & S_{n-1} \leq s<X,
\end{array}\right.
$$

where $V_{\max s i}$ is the static speed limits of the section $i$ $(i=1,2, \ldots, n)$, in $\mathrm{km} / \mathrm{h} ; S_{i}$ is the border position of section $i$, in $\mathrm{m}$; and, $s$ is the train position, in $\mathrm{m}$.

\subsubsection{Dynamic speed constraints of the following train}

The dynamic speed constraints of the following train are caused by the leading train, whose position restricts the speed of the following train for safety. The constraints are closely related to the positions of the leading train and the following train, their trip time, and the signal system. Dynamic speed constraints are very important to the safety of the train tracking. After analyzing the working principle of the four-aspect autoblock system, we formulate the dynamic speed constraints of the following train as follows: 


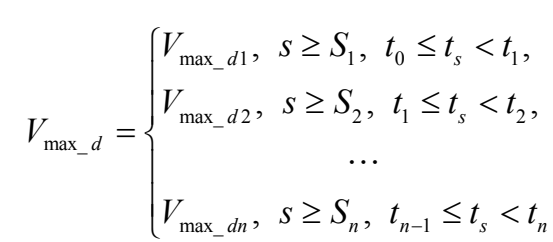

where $V_{\max \_d i}$ is the max safe speed of the following train in section $i$ during the period from $t_{i-1}$ to $t_{i}$, and $t_{i}$ is the time section border of dynamic speed constraints, which is defined dynamically by the leading train position.

When the leading train passes every signal, the data of the dynamic constraints will be recalculated according to the working principle of the four-aspect autoblock system.

\subsubsection{Constraints of shifting the train operating state}

There are three operating states for a train, i.e., motoring, coasting, and braking. The shifting rule is listed in Table 1 [8].

Table 1 Principle of shifting train operating state

\begin{tabular}{cccc}
\hline \multirow{2}{*}{$\begin{array}{c}\text { Current } \\
\text { operating state }\end{array}$} & \multicolumn{3}{c}{ Operating states to be shifted } \\
\cline { 2 - 4 } & Motoring & Coasting & Braking \\
\hline Motoring & $\circ$ & $\bigcirc$ & $\bullet$ \\
Coasting & $\bigcirc$ & $\circ$ & $\bigcirc$ \\
Braking & $\bullet$ & 0 & $\circ$ \\
\hline
\end{tabular}

०: need not shfit; ( ) : can shfit; $\bullet$ : cannot shfit

\subsection{Object function}

This problem is difficult to solve with these constraints mentioned above. In order to simplify the solving process, we introduce an external penalty function method to transform this problem to another without constraints. The object function is demonstrated as follows:

$$
\begin{aligned}
& \min E=\min \left(E_{1}+E_{2}\right), \\
& E_{1}=\int_{0}^{T} Q_{y(t)} \mathrm{d} t+\alpha\left(t_{(X)}-T\right)^{2}+\beta \int_{0}^{X} \operatorname{load}_{(s)} \mathrm{d} s, \\
& E_{2}=\int_{0}^{T} Q_{y(t)} \mathrm{d} t+\alpha\left(t_{(x)}-T\right)^{2}+ \\
& \beta \int_{0}^{X} \operatorname{load}_{(s)} \mathrm{d} s+\gamma \int_{0}^{X} \int_{0}^{T} \operatorname{load}_{(s, t)} \mathrm{d} t \mathrm{~d} s, \\
& \operatorname{load}_{(s)}=\left\{\begin{array}{l}
\delta, V_{(s)}>V_{\text {max }_{-} s}, \\
0, \text { else, }
\end{array}\right. \\
& \operatorname{load}_{(s, t)}=\left\{\begin{array}{l}
\eta, V_{(s, t)}>V_{\text {max }_{-} d}, \\
0, \text { else. }
\end{array}\right\}
\end{aligned}
$$

where $E_{1}$ is the object functions of the leading train, $E_{2}$ is the object functions of the following train, $\operatorname{load}_{(s)}$ is the penalty function of the static speed constraints, $\operatorname{load}_{(s, t)}$ is the penalty function of the dynamic speed constraints, and $\alpha, \beta$ and $\gamma$ are the factors of the trip time error, static overspeed and dynamic overspeed.

\section{Genetic algorithm}

The problem under study is a nonlinear one; thus, we use GA to solve it.

\subsection{Chromosomes}

\subsubsection{Structure of chromosomes}

As mentioned above, the control variables in (3) are the train control notch and the corresponding train position, and they must be included in the genes of the chromosomes. The structure of chromosomes is illustrated in Fig. 2, where $l_{1 i}$ and $l_{2 i}$ are the control notches of the leading train and the following train, respectively, which both include motoring (integer, greater than 0 ), coasting (0) and braking (-1); $p_{1 i}$ and $p_{2 i}$ are the positions of the two trains where their control notches are changed.

\begin{tabular}{|l|l|l|l|l|l|l|l|}
\hline$l_{11} p_{11}$ & $l_{12} p_{12}$ & $\ldots$ & $l_{1 n} p_{1 n}$ & $l_{21} p_{21}$ & $l_{22} p_{22}$ & $\ldots$ & $l_{2 n} p_{2 n}$ \\
\hline
\end{tabular}

Fig. 2 Structure of chromosomes

The length of chromosomes should be as short as possible for improving the searching speed and convergence of GA. The strategy of dividing ramps into three parts [9] is introduced here. That is, a long ramp can be divided into three parts. The train adopts the same operating mode as the previous ramp in the first part. At the beginning of the second part, the train changes the operating mode to fit the gradient of the second part. In the third part, the train changes the operating mode again to prepare for the next ramp. The real number coding strategy is also applied for the same purpose.

\subsubsection{Fitness function}

The chromosome is evaluated by its fitness value (must be positive) in GA. The larger the fitness of a chromosome is, the more likely the chromosome is selected into the next generation. From (11), the following fitness function is derived:

$$
f_{i}=1 /\left(E_{1}+E_{2}\right),
$$

where $f_{i}$ is the fitness of the chromosome $i$. The fitness function guarantees two necessary conditions for the fitness. 
First, it is positive. Second, the less energy the trains consumes, the bigger the fitness of the chromosome.

\subsection{Operators of $G A$}

\subsubsection{Selection operator}

Roulette wheel selection based on elitism strategy is adopted in this paper. The probability of each chromosome in every population is calculated by (16). Refs. [10-11] demonstrates that the GA with elitism strategy is convergent in the global searching scope with the Markov chain:

$$
p_{i}=\frac{f_{i}}{\sum_{j=1}^{N} f_{j}}=\frac{f_{i}}{f_{\text {sum }}}
$$

where $f_{\text {sum }}$ is the total fitness of the population, and $p_{i}$ is the selection probability of chromosome $i$.

\subsubsection{Crossover operator}

According to the characteristics of the problem under study, the two-point parallel crossover operator is adopted. That is, a pair of parent chromosomes selected randomly is cut off at two positions. One is located in the leading train genes, and the other in the following train genes. Then the chromosome sections are exchanged and recombined to create the new offspring chromosomes.

\subsubsection{Mutation operator}

A mutation operator based on probability is used in this article. GA mutates the chromosomes randomly based on the preset mutation probability.

\subsection{Parameters of $G A$}

The parameters of GA are listed in Table 2.

Table 2 Parameters of GA

\begin{tabular}{ccccc}
\hline Generations & Populations & Chromosomes & Probability of crossover & Probability of variation \\
\hline 100 & 1 & 50 & 0.95 & 0.05 \\
\hline
\end{tabular}

\section{Simulation}

In order to verify the effectiveness of the proposed algorithm, a simulation model was created with 1 SS8 locomotive and 18 cars. The calculation process and the parameters used here were all in conformity to Train Traction Calculation Regulation [12]. The line parameters are listed in Table 3.

Table 3 Parameters of the line

\begin{tabular}{ll}
\hline Name & Value \\
\hline Departure stop & Guangzhou \\
Destination & Guangzhoudong \\
Length & $8510 \mathrm{~m}$ \\
Standard trip time & $10 \mathrm{~m}$ \\
Signal system & Four-aspect fixed automatic \\
& block signal system \\
\hline
\end{tabular}

The profile of the line is illustrated in Fig. 3, where a speed-distance curve describes an excellent driver's operating strategy in a trip from Guangzhou to Guangzhoudong. The total energy consumption was $262.09 \mathrm{~kW} \cdot \mathrm{h}$, and the running time was $559 \mathrm{~s}$ [8].

The data of braking process is calculated and the ramps of the line are simplified before the beginning of the GA according to Train Traction Calculation Regulation [12].

Through iterative calculation of GA, the speeddistance relation of the leading train and the following train are shown in Fig. 4 and Fig. 5, respectively.

The energy consumption of the leading train is $206.84 \mathrm{~kW} \cdot \mathrm{h}$ and the trip time is $576 \mathrm{~s}$. The corresponding data of the following train are $201.79 \mathrm{~kW} \cdot \mathrm{h}$ and $562 \mathrm{~s}$. Compared with the data of [8] (single train not in a train tracking, $223.086 \mathrm{~kW} \cdot \mathrm{h}$ and $603 \mathrm{~s}$ ), the energy consumption of the leading train decreased $7.3 \%$ and the trip time decreased $4.5 \%$, while the corresponding data of the following train are $9.5 \%$ and $6.8 \%$.

The average fitness of all the generations of GA is illustrated in Fig. 6. One can see that the maximum fitness is achieved at the 65 th generation 


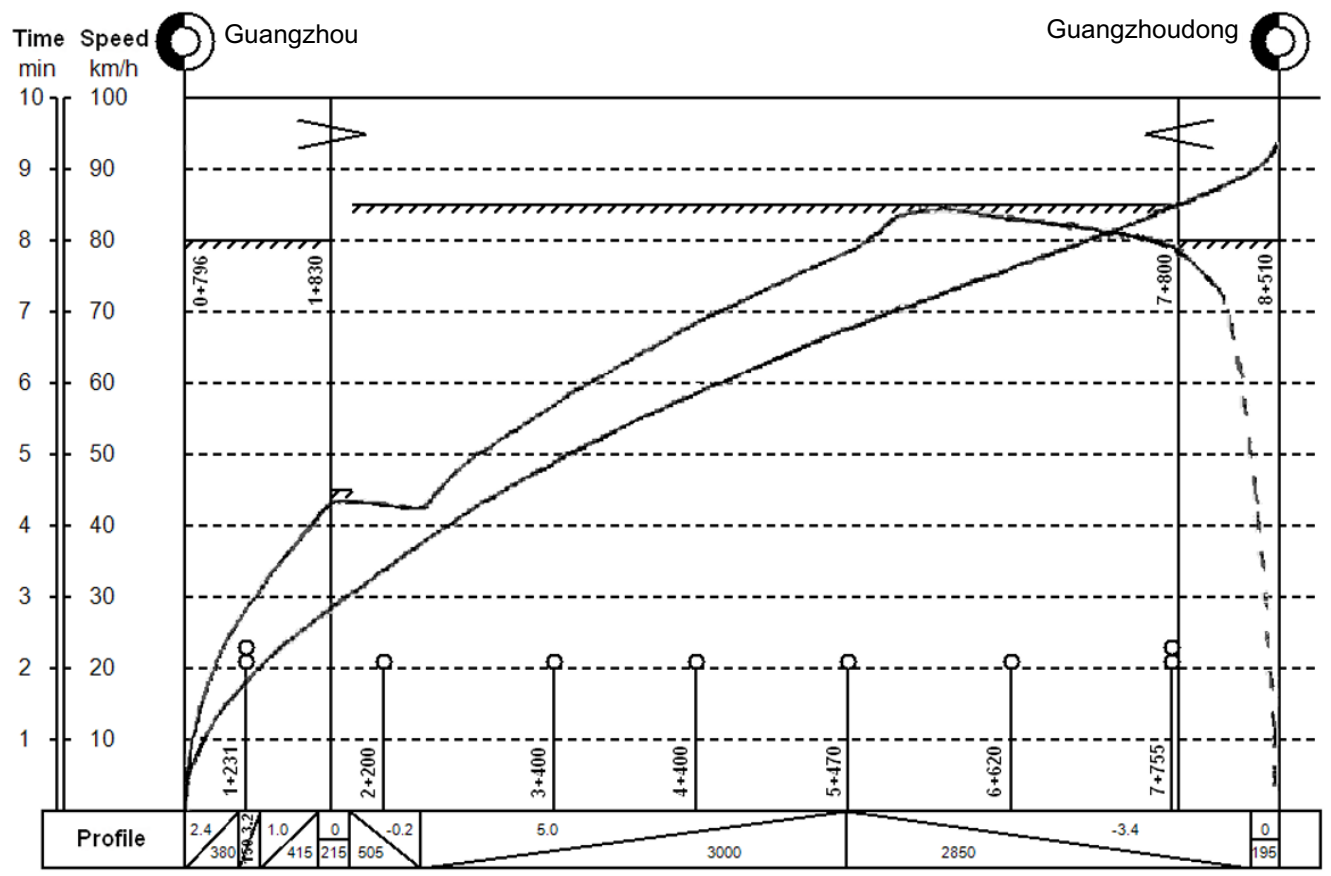

Fig. 3 Profile of the line from Guangzhong to Guangzhoudong and trajectory of the train driven by an excellent driver

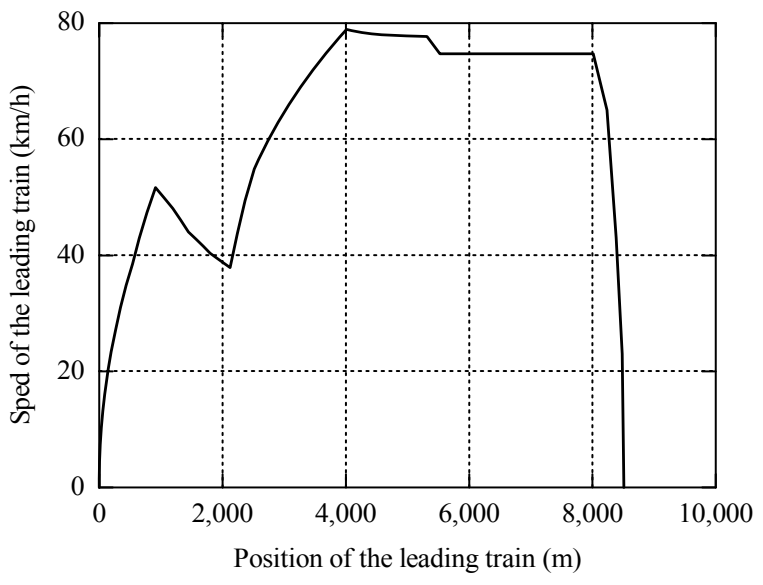

Fig. 4 Trajectory of the leading train

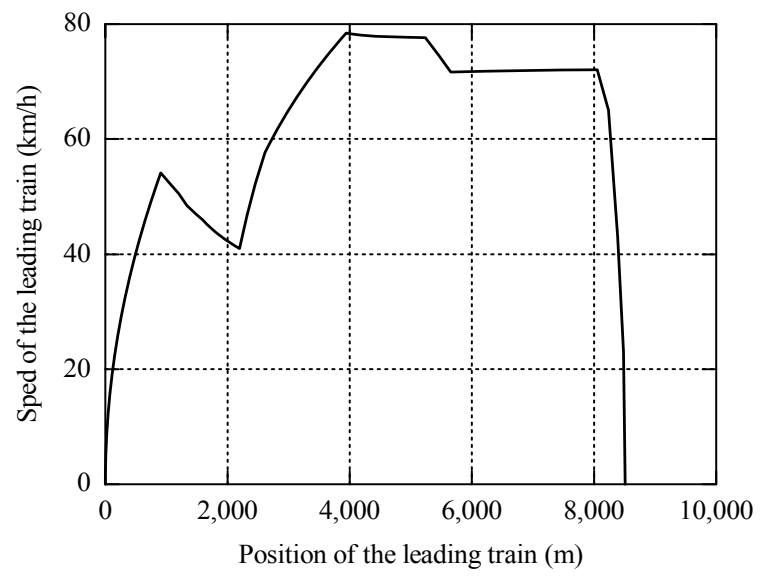

Fig. 5 Trajectory of the following train

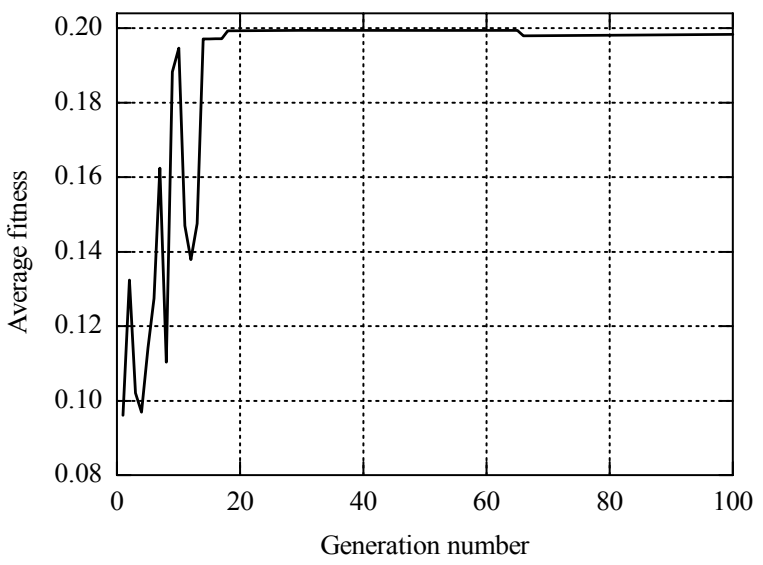

Fig. 6 Relation of GA generation and average fitness

\section{Conclusions}

(1) The static speed constraints and the dynamic speed constraints guarantee the safety of trains while running in a train tracking under the four-aspect autoblock system.

(2) It is feasible to optimize the operating strategy of the leading train and the following train with the structure of chromosomes and parallel crossover (two points) proposed in this paper.

(3) Using the real number coding and the strategy of dividing ramps into three parts, the length of chromosomes is shortened and the speed of convergence is improved.

(4) The simulation result shows that the proposed method can effectively lower the energy consumption of 
the two trains in a following operating in the premise of ensuring safety and punctuality.

\section{Acknowledgements}

This paper was supported by the National Science \& Technology Pillar Program during the Eleventh FiveYear Plan Period of China (No.2009BAG12A05).

\section{References}

[1] C.S. Chang, S.S. SIM, Optimizing train movements through coast control using genetic algorithm, IEEE Proc-Electr. Power Appl., 1997, 144(1): 65-73.

[2] J.X. Cheng, P. Howlett, Application of critical velocities to the minimization of fuel consumption in the control of trains, Automatica, 1992, 28(1): 165-169.

[3] J.X. Cheng, P. Howlett, A note on the calculation of optimal strategies for the minimization of fuel consumption in the control of trains, IEEE Trans. on Automatic Control, 1993, 38(11): 1730-1734.

[4] P. Howlett, Optimal strategies for the control of a train, Automatica, 1996, 32(4): 519-532.

[5] J.X. Cheng, J.S. Cheng, J. Song, et al., Algorithms on optimal driving strategies for train control problem, In: Proceedings of the 3rd World Congress on Intelligent
Control and Automation, Hefei: IEEE Press, 2000: 35233527.

[6] W.D. Jin, Z.L. Wang, C.W. Li, et al., Study on optimization method of train operation for saving energy, Journal of the China Railway Society, 1997, 19(6): 58-62 (in Chinese).

[7] Y.P. Fu, Research on modeling and simulations of train tracking operation and saving energy optimization [Dissertation], Beijing: Beijing Jiaotong University, 2009 (in Chinese).

[8] Q. He, Train optimized control based on genetic algorithm and fuzzy expert system [Dissertation], Chengdu: Southwest Jiaotong University, 2006 (in Chinese).

[9] Y.S. Li, Z.S. Hou, Study on energy-saving control for train based on genetic algorithm, Journal of System Simulation, 2007, 19(2): 1-4 (in Chinese).

[10] Y. Shi, S.L. Yu, Real-coded crossover operator and improved real-coded genetic algorithm, Journal of Nanjing University of Posts and Telecommunications (Natural Science), 2002, 22(2): 42-45 (in Chinese).

[11] D. Lin, M.Q. Li, J.S. Kou, On the convergence of realcoded genetic algorithms, Journal of Computer Research and Development, 2000, 37(11): 1322-1326 (in Chinese).

[12] The Ministry of Railways of The People's Republic of China, TB/T 1407-1998 Train traction calculation regulation, Beijing: China Railway Publishing House, 1998 (in Chinese).

(Editor: Junsi LAN) 